\title{
Contrôle du mode spatial des harmoniques d'ordres élévés
}

\author{
E. Mével*(2) ${ }^{(2)}$ Iercer*(1), R. Zerne*, A. L'Huillier**** Ph. Antoine** et \\ C.-G. Wahlström* \\ * Department of Physics, Lund Institute of Technology, 22100 Lund, Suède \\ ** Commissariat à l'Énergie Atomique, DSM/DRECAM/SPAM, Centre d'Études de \\ Saclay, 91191 Gif-sur-Yvette, France
}

\begin{abstract}
Résumé : Nous avons démontré que le mode spatial des harmoniques d'ordres élevés, produites par interaction non linéaire d'un laser intense et d'un jet de gaz, peut être continuement controlée. Le contrôle est obtenu en modulant spatialement le degré d'ellipticité dans la polarisation du laser incident à l'aide d'optiques biréfringentes. Les harmoniques sont émises sous forme de faisceaux annulaires de divergence ajustable.
\end{abstract}

\section{INTRODUCTION}

La génération d'harmoniques d'ordres élevés constitue une alternative des plus intéressantes parmi les nouvelles sources de rayonnement XUV cohérent. Cette technique permet d'engendrer un rayonnement XUV aux propriétés uniques jusqu'à des longueurs d'onde aussi courtes que $7 \mathrm{~nm}$. Les processus physiques mis en jeu lors de l'interaction sont désormais bien compris. Un développement naturel consiste maintenant à imaginer des méthodes permettant de manipuler ce rayonnement de façon contrôlée. Nous avons ainsi démontré expérimentalement qu'en modulant spatialement la polarisation du laser incident il est possible de contrôler le mode spatial des harmoniques [1].

\section{PRINCIPE ET DISPOSITIF EXPERIMENTAL}

Nos expériences reposent sur la forte dépendance de l'efficacité de la génération d'harmoniques en fonction du degré d'ellipticité de la polarisation du laser [2]. Le contrôle s'opère en introduisant une modulation spatiale, transverse, ajustable de l'ellipticité. Le mode spatial des harmoniques va reproduire cette modulation, puisque, d'un point de vue macroscopique, les harmoniques ne vont être engendrées de manière efficace que dans les zones de la région d'interaction oú l'ellipticité est proche de zéro (polarisation linéaire).

Le contrôle de l'ellipticité est obtenu à l'aide d'optiques biréfringentes. Le faisceau laser est polarisé à $45^{\circ}$ des axes neutres. Une lentille de quartz biréfringent focalise les composantes ordinaires et extraordinaires (polarisations croisées) en deux foyers distincts sur l'axe. Les fronts d'onde correspondant présentent des courbures différentes ce qui se traduit par une modulation transverse de l'ellipticité. Un compensateur de Babinet, composé de deux prismes de quartz, permet de modifier continuement la distribution radiale d'ellipticité en faisant varier le déphasage sur l'axe entre les fronts d'ondes.

Les harmoniques sont engendrées en focalisant avec ces optiques, au voisinage d'un jet pulsé d'argon de $1 \mathrm{~mm}$ d'épaisseur, le laser titane-saphir térawatt de Lund, opérant à $794 \mathrm{~nm}$, délivrant des impulsions d'une durée de $150 \mathrm{fs}$ et d'énergie limitée à $8 \mathrm{~mJ}$ pour nos

\footnotetext{
Adresses Présentes :

(1) Departments of Chemistry and Biochemistry, Imperial College, South Kensington, London, UK.

${ }^{(2)}$ Max Born Institut für Nichtlinieare Optik und Kurzzeitspectroskopie, Rudower

Chaussee 6, 12489 Berlin, Allemagne.
} 
expériences. Les harmoniques sont analysées dans un spectromètre XUV à réseau sphérique, en incidence quasi-normale. Leur distribution angulaire est mesurée avec un système d'imagerie comportant des galettes de microcanaux, un écran phosphore et une caméra $C C D$.

\section{RESULTATS ET CONCLUSION}

A l'aide de cette technique, nous avons pu varier continuement le mode spatial des harmoniques, depuis un profil de type gaussien jusqu'à un mode annulaire comportant un ou plusieurs anneaux de diamètres ajustables (Fig. 1). Cette observation a pu être menée jusqu'à l'harmonique $17(46 \mathrm{~nm})$.
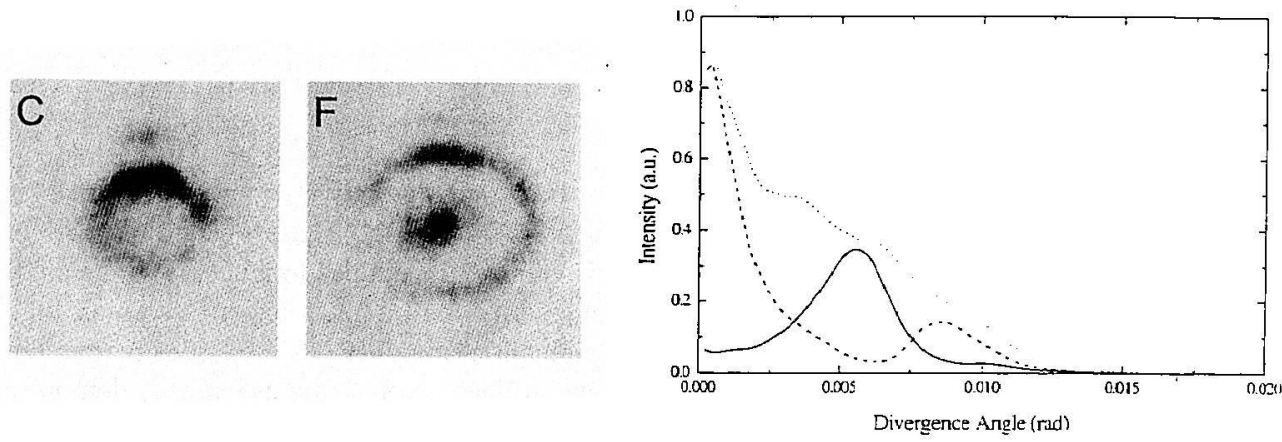

Figure 1: A gauche: images CCD du mode spatial de l'harmonique 13 pour deux positions du cunplisalleu de: Babinet $(C$ et $F)$ correspondant à des déphasages différant de $\pi / 2$. A droite : profils d'intensité moyenne correspondant aux images $C$ et $F$ (l'intensité $I(r, \theta)$ est intégrée sur l'angle $\theta$ ). La ligne pointillée représente le profil de l'harmonique 13 obtenue sans modulation (polarisation du laser parallèle à l'un des axes neutres des optiques biréfringentes).

Nous avons démontré qu'il est possible de contrôler continuement le mode spatial des harmoniques. Dans une certaine mesure, cette expérience constitue une première étape vers le contrôle temporel de l'émission des harmoniques. En modulant temporellement d'ellipticité d'une impulsion laser, il est théoriquement possible d'engendrer une impulsion XUV d'une durée de quelques centaines d'attosecondes ( 1 as $=10^{-18} \mathrm{sec}$.) [3].

\section{Remerciements}

Nous remercions le Conseil National de la Recherche Scientifique Suèdoise et le programme de la Communauté Européenne "Capital Humain et Mobilité".

\section{Références}

[1] I. Mercer, E. Mével, R. Zerne, A. L'Huillier, Ph. Antoine and C.-G. Wahlström, to be published in Phys. Rev. Lett.

[2] K. S. Budil, P. Salières, A. L'Huillier, T. Ditmire and M. D. Perry, Phys. Rev. A 48. R3437 (1993)

[3] M. Yu. Ivanov, P. B. Corkum, T. Zuo and A. Bandrauk, Phys. Rev. Lett. 74, 2933 (1995) 